\title{
Effect of number of daily trials on serial learning and performance in rats
}

\author{
STEVEN J. HAGGBLOOM, FRANK K. SHEPPARD, and ANNETTE R. HILL \\ Arkansas State University, State University, Arkansas
}

\begin{abstract}
In two experiments, rats received 40 repetitions of the decreasing series of number of $.045-\mathrm{g}$ food pellets 14-7-3-1-0 over successive runs in a runway. In Experiment 1, Group 4 received four daily trials, each consisting of one repetition of the series, and Group 1 received a single daily trial. In Experiment 2, Group 4 received four daily trials and Group 2 received two daily trials. In both experiments, learning to anticipate and to run slowly to the 0-pellet element was an increasing function of number of daily trials. At the end of Experiment 1, Group 1 was shifted to four trials per day for an additional 3 days of training but showed no improvement. This result suggests that the poor anticipation of 0 pellets in Group 1 was due to a learning deficit, not merely to a performance deficit. That is, the use of multiple daily trials appears to facilitate the learning of a serial task.
\end{abstract}

Hulse (1980) suggested that procedural differences between his experiments (e.g., Hulse \& Dorsky, 1977, 1979) and those of Capaldi and his associates (e.g., Capaldi, Blitzer, \& Molina, 1979; Capaldi \& Molina, 1979) might account for the fact that his experiments support a rule-learning model of rat serial learning and that Capaldi's results favor a stimulus-stimulus (S-S), or interitem, association model. Pattern length, the temporal interval between runs to successive pattern elements (interrun interval, or IRI), and number of daily pattern repetitions (trials) were all identified by Hulse (1980) as variables that differed between his experiments and those of Capaldi, variables that might determine whether rats learn abstract rules or S-S associations. Capaldi, Nawrocki, Miller, and Verry (1985) have presented evidence, however, that those variables probably do not determine whether rats learn rules or S-S associations.

Although the strategy that rats employ to solve serial learning problems may be independent of variables such as IRI or number of daily trials, those variables may, nevertheless, have a substantial effect on the rate of serial learning, a better understanding of which might provide clues about what is learned. Thus, for example, which of two series is learned faster does not necessarily depend on the IRI (Capaldi, Nawrocki, Miller, \& Verry, 1985), but under some conditions, the rate at which a particular series is learned is facilitated by a short IRI (Haggbloom \& Ekdahl, 1985; Roitblat, Pologe, \& Scopatz, 1983). This effect of IRI appears to be due to the rat's use of temporal cues associated with a relatively long interval between successive series presentations to phrase the series

These experiments were supported by a Faculty Research Grant to the first author from Arkansas State University. Requests for reprints should be addressed to the first author. Department of Psychology, Arkansas State University, P.O. Box 2127, State University, AR 72467-2127. [e.g., to discriminate the end of one series presentation from the beginning of the next (Capaldi, Miller, \& Nawrocki, 1985)].

In the two experiments reported here, we investigated the effect on rate of serial learning of number of daily trials, where a trial consists of one presentation of the series, a variable about which little is known. Roitblat et al. (1983) varied number of trials in a design that intentionally confounded values of that variable with differences in IRI. Based on a comparison between two experiments, they concluded that serial learning required a short IRI (cf. Haggbloom \& Ekdahl, 1985), but was not affected by number of trials per day. Capaldi, Miller, and Nawrocki (1985) found no difference between groups given one versus three trials per day, but they used a series evidently much easier to learn than that used by Roitblat et al. (1983). A series too easily learned could mask any effect of number of trials. The present experiments, like those of Roitblat et al. (1983), used the series 14-73-1-0, where the numbers represent the quantity of .045-g food pellets received on successive runs. Experiment 1 investigated the effects of one versus four trials per day, and Experiment 2 investigated the effects of two versus four trials per day, on serial learning performance.

\section{EXPERIMENT 1}

\section{Method}

Subjects. The subjects were 16 experimentally naive male rats, approximately 120 days old at the beginning of the experiment, bred in the laboratory from Holtzman stock.

Apparatus. The apparatus was a straight enclosed runway, modeled after that used by Hulse and Dorsky (1977). The runway was $92 \mathrm{~cm}$ long with a $30-\mathrm{cm}$ startbox and a $30-\mathrm{cm}$ goal area, separated from the rest of the runway by manually operated guillotine doors. The goal area contained an 11-cm niche at a right angle to the runway and an unpainted goal cup positioned against the end wall of the goal area. The goal cup was constructed by drilling a 6-cm diameter hole in a small block of wood. The inside width of the runway was $10 \mathrm{~cm}$, and the height was 
$11 \mathrm{~cm}$. The runway was painted flat black throughout. The start and goal areas were covered with hinged Plexiglas, and the runway proper was covered with hardware cloth. Raising the guillotine door between the startbox and the runway triggered a .01-sec timer, which was stopped when the rat entered the goalbox and interrupted a photobeam $20 \mathrm{~cm}$ into the goal area. Food reinforcement consisted of an appropriate number of $.045-\mathrm{g}$ Noyes pellets.

Procedure. All rats were housed individually and had free access to water at all times. Two weeks before the beginning of experimental training, the rats were placed on a food-deprivation schedule, consisting of $12 \mathrm{~g}$ of food per day, which continued throughout the experiment and was adjusted according to the weight of food pellets consumed in the runway. On Days 12-14 of deprivation, the rats were handled in squads of 2 for 2-3 min per squad. In addition to being handled on Days 13 and 14, the rats were placed in the runway and allowed to explore it for $5 \mathrm{~min}$. On both days, the goalbox was baited with 14 pellets.

Experimental training began on Day 15 of deprivation. Rats in Group 4 received four daily presentations of the 14-7-3-1-0 series for 10 days. Rats in Group 1 received a single daily presentation of the series for 40 days. The IRI was 10-15 sec for both groups. The interseries interval for Group 4 was about 10 min. Training for rats in Group 4 was staggered such that 2 rats were trained on Days 1-10, 2 more on Days 11-20, 2 more on Days 21-30, and the last 2 on Days 31-40. Training for the 8 rats in Group 1 was interspersed each day among the four repetitions of the pattern administered to the 2 rats currently being trained in Group 4. Following initial training, subjects in Group 1 were shifted to four trials per day for 3 days, and training for rats in Group 4 was discontinued.

A run was initiated by placing the rat in the startbox and, after approximately $3 \mathrm{sec}$, opening the startbox door. If the trial was not completed in $60 \mathrm{sec}$, the rat was placed in the goalbox and a time of $60 \mathrm{sec}$ was recorded for that trial. The goalbox was baited with the appropriate number of $.045-\mathrm{g}$ food pellets on reinforced trials. On nonreinforced trials, the rat was confined to the unbaited goalbox for $10 \mathrm{sec}$.

\section{Results and Discussion}

One rat in Group 4 died, and its data were discarded. Figure 1 shows the mean running time for both groups on 14-pellet and 0-pellet runs in blocks of four trials (each block is a day for Group 4). The inset shows running times for both groups to each pattern element collapsed over the last two trial blocks. The inset also shows times on Trial 1 on the last two trial blocks (days) for Group 4.

As can be seen in Figure 1, Group 4 learned to anticipate the 0-pellet element sooner and substantially better

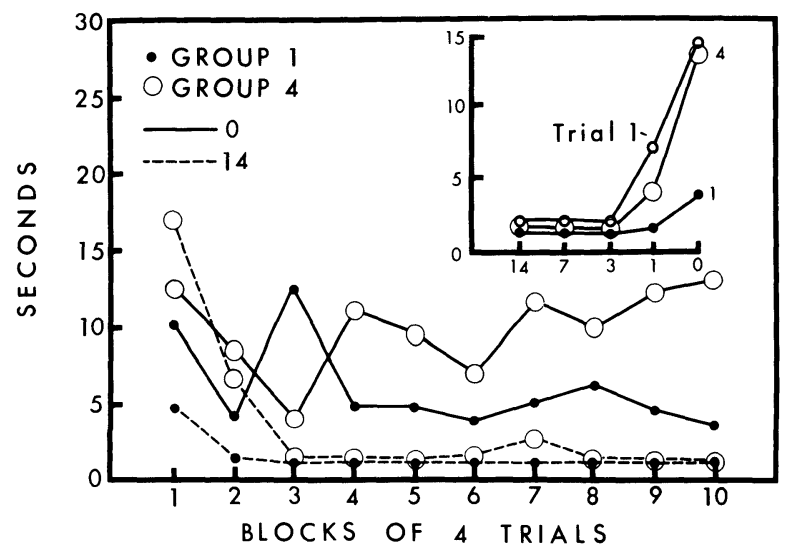

Figure 1. Running times for both groups in Experiment 1 on 14pellet and 0-pellet runs in blocks of four trials. The inset shows running times to all five elements averaged over the last two trial blocks. than did Group 1. By the end of training, group times were essentially the same to the first three pattern elements (inset), but Group 4 ran slightly slower to 1 pellet, and very much slower to 0 pellets than did Group 1 .

An analysis of variance applied to times on the last two trial blocks yielded reliable effects of groups $[F(1,13)=$ $5.83, p<.05]$, runs $[F(4,52)=14.28, p<.001]$, and runs $\times$ groups $[F(4,52)=5.36, p<.01]$. Planned comparisons showed that Group 4 ran slower than did Group 1 on Run $5[F(1,13)=25.64, p<.01]$, but that the two groups did not differ on Runs 1-4 (all $F$ s $<1$ ). Additional comparisons showed that Group 4 ran reliably slower on Run 5 than on Run $1[F(1,52)=47.78$, $p<.001]$, but the difference between running times on Run 4 versus Run 1 was not reliable $[F(1,52)=3.31]$. Group 1, on the other hand, did not run reliably slower at the end of training on Run 5 than on Run $1[F(1,52)$ $=3.30$ ]. These analyses support the observation made above that anticipation of the 0-pellet element developed faster and was better in Group 4 than in Group 1.

In order to evaluate the possibility that the superior performance of Group 4 was due to a warm-up effect, we examined behavior in that group on the first trial of the day. As can be seen in the inset in Figure 1, the performance of Group 4 on the first trial of the day was essentially the same as the average performance over all four daily trials. To determine whether the poor performance in Group 1 reflects a learning deficit or a performance deficit, that group was shifted to four trials per day for an additional 3 days of testing. The mean running times on Run 5 on those days were $2.77 \mathrm{sec}, 2.15 \mathrm{sec}$, and $2.39 \mathrm{sec}$, essentially unchanged from Phase 1 (see Figure 1). The fact that the performance of Group 1 showed no improvement over 3 days of training at four trials per day suggests that poor performance at one trial per day reflects a learning deficit, and not merely a performance deficit.

\section{EXPERIMENT 2}

Experiment 1 demonstrated that performance on a serial learning task was better in rats given four trials per day than in those given only one trial per day. Experiment 2 investigated whether the advantage to Group 4 was due simply to the experience of multiple trials per day and might be fully realized with just two daily trials. The alternative is that performance is truly an increasing function of number of trials, so that a group given four trials per day would also perform better than a group given multiple trials at the rate of two per day. In Experiment 2, Group 2 received two daily repetitions of the series 147-3-1-0 and Group 4 received four daily repetitions of the same series.

\section{Method}

Subjects. The subjects were 16 male rats, approximately 75 days old at the beginning of training, bred in the laboratory from Holtzman stock.

Apparatus. The apparatus was the same as in Experiment 1.

Procedure. All procedures prior to Day 1 of training were the same as in Experiment 1. The experiment was conducted in two replications 
with 4 rats per group in each replication. The procedures described below refer to a single replication. Training began on Day 15 of deprivation, with rats in Group 2 receiving two trials per day for 20 days. One half of the subjects in Group 4 were run on Days 1-10 and the other half of the subjects were trained on Days 11-20. Daily trials for subjects in Group 2 were interspersed among those for Group 4. The reward series and all trial administration procedures were the same as in Experiment 1.

\section{Results and Discussion}

One rat from each group died, and their data were discarded. Figure 2 shows the mean running time for both groups on 14-pellet and 0-pellet runs in blocks of four trials. The inset shows the performance of both groups on each run averaged over the last four trial blocks. As can be seen, differences between the two groups in anticipation of the 0-pellet element did not emerge until about Block 7, but the difference eventually favored better performance by Group 4 over Group 2. As can be seen in the inset, Groups 4 and 2 responded about the same to the first three pattern elements by the end of training, but Group 4 ran slightly slower to 1 pellet and substantially slower to 0 pellets than did Group 2 .

An analysis of variance applied to running times on the last four trial blocks yielded reliable effects of runs $[F(4,40)=26.89, p<.001]$ and of runs $\times$ groups $[F(4,40)=3.40, p<.05]$, but no main effects of groups $[F(1,10)=2.54]$ or replications $(F<1)$. The runs $\times$ groups $\times$ replications interaction was also not significant $(F<1)$. Planned comparisons involving group mean times over the last four trial blocks showed that Group 4 ran slower on Run 5 than did Group $2[F(1,10)=15.55$, $p<.01]$, but the groups did not differ on any other runs (all $F \mathrm{~s}<1$ ). Additional comparisons showed that Group 4 ran slower on Run 5 than on Run $1[F(1,40)=$ $71.13, p<.001]$, and also ran slower on Run 4 than on Run $1[F(1,40)=5.26, p<.05]$. Group 2 also ran slower on Run 5 than on Run $1[F(1,40)=16.61$, $p<.001]$. These analyses support the observations made

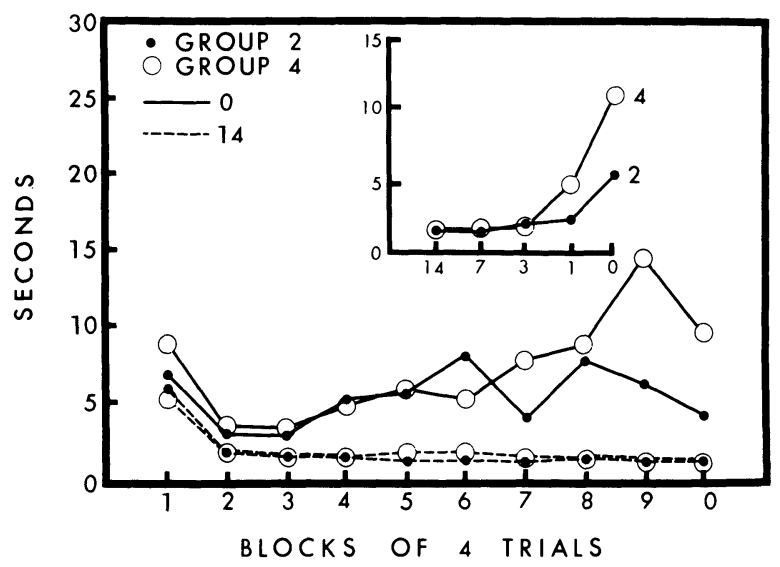

Figure 2. Running times for both groups in Experiment 2 on 14 pellet and 0-pellet runs in blocks of four trials. The inset shows running times to all five elements averaged over the last four trial blocks. above that performance on this task was better for Group 4 than for Group 1.

\section{GENERAL DISCUSSION}

The two experiments reported here indicate that learning and performance on the serial pattern 14-7-3-1-0 improve with an increase in the number of daily trials. Roitblat et al. (1983) suggested that number of daily trials was not an important variable in serial learning, but their experimental design did not permit a direct assessment of the effects of number of trials. Capaldi, Miller, and Nawrocki (1985) reported no difference in rate of learning or performance between groups, given one versus three daily trials on the series 18-1-0. However, learning occurred very rapidly on their 18-1-0 series, perhaps too rapidly for the three-trial group to have shown an advantage over the one-trial group. There might be an interaction between task difficulty and number of daily trials such that additional trials promote serial learning and performance to a greater extent as problem difficulty increases.

It is possible that the better performance of Group 4 over Group 1 in Experiment 1 was not so much a function of number of daily trials, but rather, that the performance of Group 1 might have been impaired by the very long intertrial (interseries) interval experienced by that group. Performance on a variety of discrimination problems regulated by the stimulus consequences of goal events is impaired by a long intertrial interval (Haggbloom, 1978, 1979a; Jobe, Mellgren, Feinberg, Littlejohn, \& Rigby, 1977), and there is a marked impairment of brightness differential conditioning in groups given only one trial per day (Haggbloom, 1979b). However, the better performance of Group 4 over Group 2 in the present Experiment 2, where the intertrial interval was the same for both groups, suggests that number of daily trials is the important variable.

We would emphasize that the present results are not immediately relevant to the dispute over whether rats can acquire knowledge about the structure of a series (i.e., learn a rule) and are more likely to do so with multiple trials than with single trials, as suggested by Hulse (1980), or whether, as suggested by Capaldi and his associates, serial learning in rats is regulated by S-S associations. However, an understanding of the conditions under which serial learning is promoted by multiple daily trials should contribute to our knowledge about what is learned on such tasks.

\section{REFERENCES}

Capaldi, E. J., Blitzer, R. A., \& Molina, P. (1979). Serial anticipation pattern learning in two-element and three-element series. Bulletin of the Psychonomic Society, 14, 22-24.

Capaldi, E. J., Miller, D. J., \& NaWrocki, T. M. (1985). Retention interval and intertrial interval in a serial learning or delayed discrimination task. Manuscript submitted for publication.

CAPaldi, E. J., \& Molina, P. (1979). Element discriminability as a determinant of serial pattern learning. Animal Learning \& Behavior, 7, 318-322.

Capaldi, E. J., Nawrocki, T. M., Miller, D. J., \& Verry, D. R. (1985). An examination into some variables said to affect serial learning. Animal Learning \& Behavior, 13, 129-136.

HAGGBLOOM, S. J. (1978). Intertrial interval effects on internal stimulus control of behavior in brightness differential conditioning. Learning \& Motivation, 9, 347-358.

HAGGBLOOM, S. J. (1979a). The differential reinforcement of rewardproduced and response-produced stimuli. Learning \& Motivation, 10, 364-381.

HAGGBLOOM, S. J. (1979b). Effects of a 24-hour intertrial interval on successive differential conditioning and simultaneous negative contrast. American Journal of Psychology, 92, 537-546.

HAGGBloOM, S. J., \& EKDAHL, M. W. (1985). Effects of interrun interval on serial learning. Animal Learning \& Behavior, 13, 98-102.

Hulse, S. H. (1980). The case of the missing rule: Memory for reward vs. formal structure in serial-pattern learning by rats. Animal Learning \& Behavior, 8, 689-690.

Hulse, S. H., \& DorsKy, N. P. (1977). Structural complexity as a de- 
terminant of serial pattern learning. Learning \& Motivation, 8, 488-505.

HulSE, S. H., \& DoRSKy, N. P. (1979). Serial pattern learning by rats: Transfer of a formally defined stimulus relationship and the significance of nonreinforcement. Animal Learning \& Behavior, 7, 211-220.

Jobe, J. B., Mellgren, R. L., Feinberg, R. A., Littlejohn, R. L. \& RIGBY, R. L. (1977). Patterning, partial reinforcement, and N-length as a function of reinstatement of retrieval cues. Learning \& Motivation, 8, 77-97.

Roitblat, H. L., Pologe, B., \& Scopatz, R. A. (1983). The representation of items in serial position. Animal Learning \& Behavior, 11, 489-498.

(Manuscript received for publication October 8, 1985.) 